\title{
Bossuet face à La Vallière : la topique de la vanité, entre enjeux religieux et enjeux politiques
}

Dans le Sermon sur l'honneur du monde, Bossuet prononce un violent réquisitoire contre les vanités terrestres, appelées "à un tribunal où [leur] condamnation est bien assurée » (1927, p. 342). Dans ce procès, « les sceptres rejetés, l'honneur méprisé, toute la gloire du monde anéantie font le plus grand ornement [du] triomphe [du Christ] " (Bossuet, 1927, p. 341). Devant un auditoire composé de courtisans, mais aussi du Prince de Condé, l'Aigle de Meaux ne condamne pas seulement les vanités, mais tous ceux qui s'y complaisent - en définitive, son auditoire lui-même. Aussi les motifs de la vanité et du mépris du monde chez Bossuet ne relèvent-ils pas seulement d'une construction topique, mais aussi d'une construction énonciative. A priori, ses sermons relèvent du discours religieux, mais - nous allons le voir le contexte de 1662 rend le cadre énonciatif plus complexe.

C'est cette année-là que Bossuet prêche pour la première fois au Louvre, devant la Cour. Ce cycle de sermons est connu pour de saisissantes images de la vanité. Or 1662 est aussi la date de la relation de Louis XIV avec Louise de La Vallière. L'audacieux prédicateur profite donc de l'occasion qui lui est donnée de parler au roi pour condamner publiquement, de façon à la fois sous-entendue et claire, l'attitude immorale du monarque, qui doit être un exemple de vertu pour ses sujets et pour le reste des hommes. Puis, en 1675, à l'occasion de l'entrée de La Vallière au Carmel, M. de Meaux montera en chaire une nouvelle fois pour vilipender la vanité devant la postulante, mais aussi devant la reine.

Dans ce parcours du Carême du Louvre (1926a, p. 150-399) au Sermon pour la profession de Madame de La Vallière (1926b, p. 32-58), Bossuet ne parle pas seulement

- Nicolas Pelleton - agrégé de lettres modernes, doctorant à l'Université Clermont Auvergne, Clermont-Ferrand, France. Adresse pour correspondance : nicolas.pelleton@wanadoo.fr 
comme un moraliste catholique, mais aussi comme un conseiller politique, ce qui modifie la valeur des motifs de la vanité, hérités de l'Ecclésiaste. Grâce à l'analyse des trois pôles de l'énonciation, nous allons montrer que les modalités par lesquelles les motifs de la vanité et du mépris du monde sont construits oscillent sans cesse pour reprendre la terminologie de Dominique Maingueneau ${ }^{1}$ - entre scène dénonciation religieuse et scène d'énonciation politique : l'enargeia et la topique baroques de Bossuet sont aussi le signe de son audace politique.

\section{De la cour : prêcher le mépris des vanités dans un espace de mondanités}

En 1662, l'Aigle de Meaux prêche dans la chapelle de l'Oratoire du Louvre. Cet espace référentiel et physique appartient à un lieu plus vaste, la cour, qui est par excellence celui de la vanité et de l'ambition. Or c'est précisément ce cadre, apparemment inapproprié au mépris des biens de ce monde, que l'orateur va utiliser pour donner à sa condamnation de l'attitude du roi un sens résolument politique.

\subsection{Une rhétorique du desengaño}

Le mot portugais desengaño, qui signifie "déception », désigne une forme de désillusion sur le monde et sur les biens terrestres. Bossuet en fait une arme de sa campagne contre la liaison du roi et de La Vallière. Ainsi, il joue sur la représentation sociale de la femme au XVII' siècle :

Que vous dirai-je maintenant, Mesdames, du temps infini qui se perd dans de vains ajustements ? [...] on s'en joue [du temps], on le prodigue sans mesure jusqu'aux cheveux, c'est-à-dire, la chose la plus nécessaire à la chose la plus inutile. (Bossuet, 1926a, p. 353)

Au XVII siècle, la féminité est associée à la légèreté. De façon indirecte, La Vallière devient donc un ennemi public, qui précipite la cour dans le superflu. Par ailleurs, on peut considérer la mention de l'inutilité des cheveux comme figuration biaisée d'une prise de voile. Au contraire, dans le Sermon pour la profession de La Vallière, Bossuet dresse un portrait de l'âme pécheresse, qui est « défigurée " (1926b, p. 38), mais dont le salut est assuré par la prise de voile (1926b, p. 57-58).

Les questions rhétoriques font aussi partie de la rhétorique du desengaño : "Qu'est-ce que cent ans, qu'est-ce que mille ans, puisqu'un seul moment les efface? ? (Bossuet, 1926a, p. 267) "Mais hélas ! que nous profite cette dignité ? (Bossuet, 1926a, p. 279). En 1662, la rhétorique du desengaño sert à convaincre les amants de rompre. Cela étant, on retrouve cette rhétorique dans le Sermon pour la profession

1. La « scène englobante » est celle sur laquelle « il faut se placer pour [...] interpréter » le discours (Maingueneau, 2004, p. 191). 
de La Vallière : "Elle n'est pas plus heureuse en jouissant des plaisirs que ses sens lui offrent ; [...] " (Bossuet, 1926b, p. 41). Le motif de la légèreté féminine y est lui aussi à nouveau présent : «le Saint-Esprit a voulu descendre dans un dénombrement exact de tous les ornements de la vanité ", c'est-à-dire " les rubans, et les broderies ", «vaines couvertures qui ne cachent rien » (Bossuet, 1926b, p. 49). Or en 1675, la rhétorique du desengaño ne sert plus la répudiation de La Vallière, puisqu'à cette date, celle-ci prend définitivement le voile. Le but de cette rhétorique, moral et théologique (à première vue), est de dresser un tableau de la pénitence de l'âme ; mais du point de vue de l'efficacité pragmatique, il s'agit d'affirmer de façon spectaculaire le triomphe politique de l'Église.

\subsection{Le style prophétique}

À l'opposé du desengaño, Bossuet a recours à un style que l'on peut qualifier de prophétique. Avec tout ce qu'il comporte de solennel, le style prophétique est propre à susciter la terreur. Pour le prédicateur, le «silence » de Dieu « est une voix publique qui invite tous les pécheurs à se reconnaître " (Bossuet, 1926a, p. 173). Dans la mesure où la liaison de Louis XIV et de La Vallière était de notoriété publique (CagnatDebœuf, 2001, p. 15-16), on peut considérer que cette " voix publique » renvoie au discours que les contemporains de l'affaire tenait sur celle-ci. Ainsi, M. de Meaux nourrit d'autres discours son propre discours pour rendre plus virulent son propos.

Le « ô » exclamatif fonde le style prophétique de Bossuet : «O calamité de nos jours! » (1926a, p. 213), " ô dénûment d'un cœur chrétien! " (1926a, p. 296), « O fragile appui de notre être ! ô fondement ruineux de notre substance !» (1926a, p. 270). L'impératif a la même fonction : "Perds-toi dans l'abîme du saint amour " (Bossuet, 1926a, p. 294). Dans le Sermon pour la profession de La Vallière, l'impératif est le mode de l'exécution des desseins de Dieu : «Et vous, ma Sour, [...] allez à l'autel $;[\ldots]$ victime de la pénitence, allez achever votre sacrifice : [...] " (Bossuet, 1926b, p. 57). La parole ecclésiastique occupe donc la fonction du glaive qui immole la victime, en présence de la reine, à la fois caution politique et garante du triomphe de la religion.

Une forme de menace est nécessaire, qui rappelle au roi ses devoirs envers ses sujets et envers Dieu ; à cet égard, le prédicateur devient un "spécialist [e] religieux " compétent dans la " gestion des biens de salut " (Bourdieu, 1971, p. 304) et a ainsi une fonction politique. Le futur simple revêt des qualités évocatoires fortes :

Dans cette crainte, dans cette épouvante, dans ce silence universel de toute la nature, avec quelle dérision sera entendu le raisonnement des impies, qui s'affermissaient dans le crime en voyant d'autres crimes impunis ! (Bossuet, 1926a, p. 227)

Face au dérèglement qui saisit la cour et la tête de l'État, Bossuet utilise à la fois le futur simple et une diatypose dont l'efficacité est redoutable : la fin de toute vie se donne alors comme la conséquence inéluctable des "crimes" des grands. 
Le « silence » est patent dans les péroraisons, qui ouvrent sur un temps de méditation, en particulier pour le roi :

Puisse Votre Majesté avoir bientôt le moyen d'assouvir son cœur de ce plaisir vraiment chrétien et vraiment royal de rendre ses peuples heureux ! [...] c'est ce qui comblera Votre Majesté d'une gloire si accomplie, qu'il n'y aura plus rien à lui désirer que la félicité éternelle [...]. (Bossuet, 1926a, p. 399)

De façon implicite, le prédicateur voit dans la rupture du roi et de sa maîtresse le « moyen " par lequel Louis XIV reviendra pleinement à ses devoirs envers «ses peuples » : c'est la condition sine qua non de son salut, de sa "gloire » future.

L'utilisation politique et publique du style prophétique passe aussi par l'amplificatio et par le sublime :

Ce sera sans doute un grand spectacle, quand Celui qui est assis sur le trône d'où relève tout l'univers, [...] prononcera du haut de son trône, à la fin des siècles, qu'il va renouveler toutes choses [...]. (Bossuet, 1926b, p. 34)

Ce moment solennel de l'entrée de La Vallière au Carmel est garanti politiquement par l'adresse initiale à la reine, et scellé par une forme de sublime qui rappelle l'Apocalypse. Le style prophétique cristallise alors des enjeux à la fois théologiques et politiques : la "gloire » du monde est vaine, seule importe l'éternité. L'enargeia du style prophétique a pour fonction d'attiser la « haine publique » (Bossuet, 1926a, p. 180) contre la maitresse du roi et de prêcher la pénitence.

\subsection{Un parcours de pénitence imposé à l'auditeur}

Dans le Carême du Louvre, la vanité est mise en regard du jugement du passé, du présent et du futur grâce à la construction du cadre spatio-temporel de l'énonciation : le recours à la syllepse ménage un élargissement de l'espace et du temps historiques des discours. Dès l'ouverture du Carême du Louvre, le lexème " Temple » est utilisé comme catalyseur topographique et chronographique, en ce sens qu'il ouvre le moment historique de la prédication sur les temps bibliques, et lie de cette manière le champ théologique à ce problème politico-moral qu'est la liaison du roi et de $\mathrm{La}$ Vallière :

C’est pour commencer ce mystère que Jésus entre aujourd'hui dans le Temple, non pour s'y faire voir avec majesté comme le Dieu qu'on y adore, mais pour se mettre en la place de toutes les victimes qu'on y sacrifie : [...] Et c'est tout le mystère de cette journée. (Bossuet, 1926a, p. 158)

L'utilisation du lexème "Temple » renvoie au passage de saint Luc qui narre la Présentation de Jésus au Temple, choisi par Bossuet comme " texte » support 
du sermon (1926a, p. 151); or le prédicateur a également recours à ce terme pour désigner un lieu de culte chrétien ${ }^{2}$. Cette syllepse implicite met en tension le temps biblique et le temps présent (grâce aux déictiques temporels « aujourd'hui » et « cette journée »). La situation historique est ainsi déconstruite par la dialogisation du texte biblique. Cette vertigineuse construction énonciative a pour fonction de mettre à mal tout amour des biens terrestres et éphémères, en annulant de façon spectaculaire les vicissitudes terrestres dont la royauté est atteinte. L’exégèse biblique se fait donc ici consilium politique.

En définitive, M. de Meaux doit concilier la nécessité théologique de prêcher le mépris des biens du monde, et l'obligation politique et sociale de le faire devant un auditoire enclin aux mondanités, comme dans l'exorde du Sermon sur la mort : «Me sera-t-il permis aujourd'hui d'ouvrir un tombeau devant la cour, et des yeux si délicats ne seront-ils point offensés par un objet si funèbre? » (Bossuet, 1926a, p. 262) Grâce à la rhétorique de la prétérition, l'orateur cherche à se rendre accommodant avec ses auditeurs, avant de leur rappeler leur baptême : « Je ne pense pas, Messieurs, que des chrétiens doivent refuser d'assister à ce spectacle avec Jésus-Christ » (Bossuet, 1926a, p. 262) ; le mot «chrétiens » a ici une fonction argumentative. Cette forme d'anti-rhétorique est révélatrice des biais énonciatifs auxquels doit recourir l'orateur.

\section{Du prédicateur : biais et ruses de l'énonciation}

La position de l'Aigle de Meaux est ambiguë : il doit rester prudent dans sa façon de parler au roi, tout en l'affrontant, car il appartient à la fois au champ religieux et au champ politique. Dès lors, les Écritures ne sauraient être une protection parfaite dans la mission de Bossuet. Son discours ecclésiastique et religieux utilise la métaphore à la fois comme arme défensive et comme arme offensive.

\subsection{La rhétorique judiciaire}

Sous l'Ancien Régime, les pouvoirs législatif et exécutif appartiennent au roi, mais également le pouvoir judiciaire. Or dans le Carême du Louvre et le Sermon pour la profession de La Vallière, Bossuet ne représente pas Louis XIV comme un exécuteur judiciaire, mais il l'accuse de mettre en danger la monarchie par sa liaison avec La Vallière. La rhétorique judiciaire de l'orateur cristallise alors des enjeux à la fois théologiques et moraux. L'isotopie du crime est le stylème le plus patent de la rhétorique judiciaire bossuétiste. L'oxymore " ô délices criminelles » (Bossuet, 1926a, p. 396) montre la corruption des mœurs, la corruption des « choses qui de leur nature seraient innocentes" (Bossuet, 1926a, p. 198). Bien que le mot " innocentes » actualise ici le sens de "bénignes ", "vénielles ", le style de l'Aigle de Meaux est résolument judiciaire dans ce passage. Par une expolition composée de substantifs

2. C'est par exemple le cas dans l'Oraison funèbre du Prince de Condé. 
abstraits, le prédicateur dresse le tableau des actes du mauvais riche, c'est-à-dire " ses adultères ", « ses rapines ", « ses violences » (Bossuet, 1926a, p. 198) : le pluriel de concrétisation des abstraits rend effroyables ces actions - par nature "innocentes " -, qui se résorbent dans le groupe nominal singulier « son crime». La condamnation du mauvais riche est sans appel et renvoie à ses excès et à sa vanité : "Te voilà donc, ô homme du monde, attaché à ton propre bien avec un amour immense !» (Bossuet, 1926a, p. 198). M. de Meaux active la dialogisation de ce tableau du mauvais riche, en en faisant une figuration du roi :

En quels antres profonds s’étaient retirées les lois de l'humanité et de la justice, que David savait si parfaitement, lorsqu'il fallut lui envoyer Nathan le prophète pour les rappeler en sa mémoire? (Bossuet, 1926a, p. 183)

Ici, David, roi biblique par excellence, renvoie à la personne de Louis XIV. Dès lors, Bossuet devient un nouveau Nathan qui rappelle au souverain ses devoirs.

Aussi l'orateur doit-il endosser l'ethos du conseiller politique. La scénographie du Sermon sur la Passion de Notre-Seigneur s'appuie sur une interprétation de ce qu'est un testament :

Il y a dans un testament trois choses considérables : on regarde en premier lieu si le testament est bon et valide; on regarde en second lieu de quoi dispose le testateur en faveur de ses héritiers ; et on regarde en troisième lieu ce qu'il leur ordonne. (Bossuet, 1926a, p. 378)

Le mot «testament " renvoie au testament que Jésus a "scellé " par sa mort (Bossuet, 1926a, p. 377). Or le terme est ici envisagé dans son acception juridique : la scénographie judiciaire retenue par Bossuet devient le maitre-étalon à partir duquel vont pouvoir être jugées les actions du roi. Les « décrets éternels » (Bossuet, 1926a, p. 290), les « décrets du Père " (Bossuet, 1926a, p. 291) menacent le roi : " puisque Dieu redouble ses fléaux, il faut redoubler les secours " (Bossuet, 1926a, p. 398), dit l'orateur au roi ; l'aspect inchoatif du verbe « redoubler » révèle que l'ordre politique est troublé par une menace divine, et qu'à cet égard la rupture du roi et de sa mâ̂tresse n'est pas seulement une nécessité religieuse, mais une nécessité politique.

En définitive, grâce à la rhétorique judiciaire, la parole sermonnaire devient un espace qui congédie le monde et ses vanités en s'appuyant paradoxalement sur le monde: " Esprit saint, [...] je vous ai préparé les voies en prêchant votre parole », dit Bossuet dans le Sermon pour la profession de La Vallière (1926b, p. 57). La valeur métadiscursive de cette phrase actualise sa portée religieuse ; mais l'invocation au Saint Esprit est un leurre énonciatif, puisqu'elle est destinée à la reine, à la cour et La Vallière, qui voit sa condamnation proclamée au monde vain qu'elle quitte. Tout discours théologique relève aussi du politique. 


\subsection{Métamorphoses du couple royal en allié}

La figure de la reine est convoquée par Bossuet, qui s'en fait une alliée. Pour autant, grâce à la métaphore de la royauté, le roi lui-même devient pour l'Église un allié dans ses actions pour faire répudier La Vallière. La figure royale cristallise donc le sens à la fois religieux et politique donné à la topique de la vanité.

\subsubsection{Le roi}

Selon l'orateur, le pouvoir royal tient à deux principes : « se commander [...] soimême " et " avoir autorité sur les autres " (Bossuet, 1926a, p. 250) ; il s'agit même que le roi se commande pour avoir autorité sur les autres. Cet impératif moral et politique de la maîtrise des passions est dicté à Louis XIV par la métaphore du gouvernement et de la politique, qui lui renvoie sa propre image : « O prince! c'est à vous qu'on parle " (Bossuet, 1926a, p. 183). Pour le prédicateur, ce qui menace l'exercice même du pouvoir royal, c'est que le roi soit dominé par des " péchés régnants " (1926a, p. 200). L'adjectif « régnants » construit une métaphore qui montre que les péchés deviennent pleinement actifs dans le cœur du roi. Ainsi, si les péchés dominent le roi, le roi ne peut plus commander ni être commandé.

Appliquée au roi, la métaphore du gouvernement devient réversible et structurante, parce que ce n'est pas le monarque qui est le vrai roi, mais Dieu : «cette sublime politique qui régit le monde " (Bossuet, 1926a, p. 220). C’est Dieu lui-même qui récompense les hommes de bien : «Dieu nous couronnera en son temps par la communication de son pouvoir " (Bossuet, 1926a, p. 248-249). Grâce au verbe "couronner » conjugué au futur simple, qui construit la métaphore de la royauté, l'orateur entend rappeler à Louis XIV que le pouvoir royal est la récompense d'un comportement exemplaire, et non un droit. La métaphore de la couronne permet de répondre à l'énigme du cœur humain (Bossuet, 1926a, p. 277 ; 1926b, p. 46), et de saisir dans ces reflets du divin vers l'humain et de l'humain vers le divin l'essence de Dieu.

\subsubsection{La reine}

En 1675, La Vallière entre au Carmel : elle et son ancien amant sont séparés. Louis XIV est absent lors de la profession. Or Marie-Thérèse d'Autriche, son épouse, est présente ; c'est à elle que Bossuet s'adresse dans l'exorde. Le statut énonciatif de celleci revêt une fonction politique, revendiquée par le prédicateur grâce à la mention de son titre de reine: "Madame, voici un objet digne de la présence et des yeux d'une si pieuse reine " (Bossuet, 1926b, p. 34). Le prédicateur poursuit en expliquant la présence de la reine :

Votre Majesté ne vient pas ici pour apporter les pompes mondaines dans la solitude : son humilité la sollicite à venir prendre part aux abaissements de la vie religieuse; et il est juste que, faisant par votre état une partie si considérable des grandeurs du monde, vous assistiez quelquefois aux cérémonies où on apprend à les mépriser. (Bossuet, 1926b, p. 34) 
Cela étant, cette explication n'est pas satisfaisante : elle qui était qualifiée de "pieuse » devient un symbole de la gloire et des vanités du monde ! En fait, M. de Meaux procède à des glissements paradigmatiques : les " grandeurs du monde " sont moins l'expression des vanités que celle de l'autorité politique et de la grandeur de la monarchie. Le mépris des biens du monde est assuré : le triomphe de l'Église sur Louis XIV et son ancienne maîtresse est doublement consacré par la présence de la reine, d'un point de vue politique et d'un point de vue religieux. Le vocabulaire de la vue participe à la métamorphose des "pompes mondaines " en " solitude " : le point de vue de Marie-Thérèse d'Autriche devient le prisme grâce auquel les vanités semblent paradoxalement s'annihiler au regard du monde lui-même.

\section{De la postulante : une présence-absence}

\subsection{Les modalités de l'effacement énonciatif}

Si les allusions à la liaison de Louis XIV et de La Vallière sont repérables dans le Carême du Louvre, l'affaire n'est jamais évoquée directement. Lors de la profession de l'ancienne maîtresse du roi, la présence directe de celle-ci est également limitée. Or le triomphe de la religion sur la vanité doit être humble : l'énonciation actualise à la fois cette humilité et la volonté de faire advenir pleinement le discours religieux dans le champ politique.

\subsubsection{La métonymie}

Du fait de sa valeur de trope, la métonymie est propre à dire à la fois le changement de statut de La Vallière, ancienne maîtresse de Louis XIV devenue Louise de la Miséricorde, et cet état de présence-absence qui est le sien dès le Carême $d u$ Louvre. S’adressant à la postulante le jour de sa profession, Bossuet déclare :

Considérez ensuite une âme qui, après s'être ainsi égarée, commence à revenir sur ses pas ; qui abandonne peu à peu tout ce qu'elle aimait, et qui, laissant enfin tout au-dessous d’elle, ne se réserve plus que Dieu seul. (1926b, p. 36-37)

Si La Vallière est sujet du verbe principal de la phrase ("Considérez »), on peut considérer que le COD de ce même verbe désigne par métonymie La Vallière ; les trois propositions relatives adjectives qui suivent évoquent de façon symbolique et condensée son parcours de la cour au Carmel. La cadence majeure de la phrase accroît la tension entre la deuxième personne du pluriel et le groupe nominal COD, qui semblent désigner d'un point de vue référentiel le même objet : la prise de parole du prédicateur anéantit pour la postulante tout amour possible du monde, et la négation restrictive et l'adjectif « seul » consacre cette disparition, mais aussi l'anéantissement de toutes les qualités individuelles de Louise de la Miséricorde. 


\subsubsection{L'article indéfini}

Dans le Carême du Louvre et le Sermon pour la profession de la Vallière, le style de Bossuet non seulement exprime l'anéantissement de l'individu, mais aussi participe pleinement à cette destruction. En 1662, l'affaire de la liaison du roi et de La Vallière est dans tous les esprits, et l'Église y voit un péché de Louis XIV devant Dieu. Aussi la parole du prédicateur passe-t-elle par le recours à l'implicite, fondé notamment sur le recours à l'article indéfini. Il s'agit pour Bossuet de faire comprendre sans dire ; l'article indéfini a une valeur de prétérition, comme dans le Sermon sur l'intégrité de la pénitence:

C'est à un cœur chrétien qu'il veut faire sentir ses tendresses, c'est dans un cœur chrétien qu'il veut trouver la correspondance, et ce n'est que d'un cœur chrétien que peut sortir le rebut qui l'afflige et qui le contriste. (1926a, p. 329-330; je souligne)

L'article indéfini pose l'existence de l'objet : à cet égard, il implique une saisie précoce de l'objet ${ }^{3}$. Pour autant, dans la situation historique précise et dans le contexte de l'affaire de la liaison du roi et de La Vallière, on peut aussi considérer que l'objet est indéterminé mais spécifié par le contexte : dans ce cas, la saisie est tardive. L'emploi de l'article indéfini est donc traversé par une tension sur laquelle s'appuie l'implicite : conscient du caractère potentiellement dangereux de ses propos, Bossuet adapte l'énonciation aux circonstances ; pour triompher d'un point de vue théologique et politique, le discours religieux est alors tenu de s'adapter à la scène d'énonciation publique.

\subsection{Métamorphoses et anamorphoses}

Dans le Carême du Louvre et le Sermon pour la profession de La Vallière, la double utilisation, religieuse et politique, de la topique de la vanité implique des glissements paradigmatiques, actualisés dans le style de Bossuet : il s'agit paradoxalement de rendre visible ce que la religion tend à dissimuler, et de résoudre ce paradoxe en appuyant l’énonciation sur un jeu métamorphique et anamorphique.

\subsubsection{Perspectives déformantes}

Le XVII ${ }^{e}$ siècle est une époque de découvertes et d'innovations scientifiques, notamment dans le domaine de l'optique, comme en témoignent par exemple les travaux de Descartes et de Le Nôtre. S'il reconnaît n'être " pas de ceux qui font grand état des connaissances humaines » (Bossuet, 1926a, p. 271), l'Aigle de Meaux prend actes de ces nouveautés. Aussi fait-il une utilisation métaphorique des phénomènes optiques afin de révéler sur la scène politique la vanité des mondanités et les vérités de la théologie et de la religion.

3. Dans la psychomécanique de G. Guillaume, «l'article va servir au sujet parlant à régler létendue conceptuelle du nom dans le passage de la Langue au Discours » (Joly, 2011, \$9). 
Les changements paradigmatiques que Bossuet fait subir à la notion de vanité prennent toute leur épaisseur grâce à la représentation du monde comme dispositif optique. L'orateur compare "la disposition des choses humaines [...] à certains tableaux, que l'on montre assez ordinairement dans les bibliothèques des curieux comme un jeu de la perspective » (Bossuet, 1926a, p. 220). Il ajoute :

C'est, ce me semble, Messieurs, une image assez naturelle du monde, de sa confusion apparente et de sa justesse cachée, que nous ne pouvons jamais remarquer qu'en le regardant par un certain point que la foi en Jésus-Christ nous découvre. (1926a, p. 220)

Le dispositif énonciatif fonctionne comme ce « jeu de la perspective» : en donnant un sens à la fois politique et religieux à la cérémonie de la profession de La Vallière, le prédicateur modifie par un principe anamorphique la perception des mondanités, et change par un principe métamorphique l'image même de La Vallière. Ce double phénomène revêt une valeur argumentative forte, puisqu'il s'agit de mener les auditeurs au mépris du monde : de la « confusion apparente " naît l'ordre, à la fois cosmique et politique. La cour donc est vaincue, et le Carmel devient l'espace de la vérité.

Par opposition à la copia de la cour et des mondanités, le Carmel est un lieu de dépouillement. Grâce au syntagme prépositionnel locatif « dans la solitude » (1926b, p. 34), Bossuet concrétise et spatialise une caractéristique propre au Carmel, au moment solennel où l'ancienne maîtresse royale devient Sœur Louise de la Miséricorde. Aussi est-ce la notion même de vanité qui change de paradigme et de sens : alors que la vanité de la cour renvoyait au caractère illusoire et inutile de toutes choses, la vanité du Carmel prend la mesure de l'illusion afin de faire entendre à la pénitente le langage de Dieu.

\subsubsection{La métaphore du vêtement}

La représentation du jugement divin qui menace les pécheurs implique des changements sémantiques et paradigmatiques, par lesquels ce qui était corruption devient renouvellement, et ce qui était dysphorique devient euphorique. Cette menace est mise en perspective par Bossuet dès l'exorde du sermon inaugural du Carême du Louvre:

[...] nous verrons le Fils unique et bien-aimé qui prie son Père et son Dieu qu'il puisse porter tous nos crimes, et le Père en même temps qui les lui applique si intimement que le Fils de Dieu parât tout à coup revêtu devant Dieu de tous nos péchés et, par une suite nécessaire, investi de toute la rigueur de ses jugements, percé de tous les traits de sa justice, accablé de tout le poids de ses vengeances. (1926a, p. 152)

En s'appuyant sur le style prophétique, les motifs de la vanité offrent à l'auditeur la vision de la menace du jugement final, par lequel le monde terrestre et matériel sera détruit. La mention des « crimes" des hommes rend abstrait le motif pictural 
du portement de croix, qui devient de façon soudaine et spectaculaire un embrayeur métaphorique : «le Fils de Dieu paraît tout à coup revêtu devant Dieu de tous nos péchés ». Le recours à la métaphore du vêtement associe une représentation à la fois concrète et abstraite de l'anéantissement attendu du monde. Par cette présentification violente, Bossuet en appelle à la haine du péché et du monde.

Si en 1662 le vêtement a une structure et une fonction métaphoriques, le Sermon pour la profession de La Vallière reprend ce motif dans un sens littéral et référentiel, qui est celui de la vêture : "Le sacré pontife vous attend avec ce voile mystérieux que vous demandez. Enveloppez-vous dans ce voile : vivez cachée à vous-même aussi bien qu'à tout le monde; [...] » (Bossuet, 1926b, p. 57-58). Le motif du secret n'a pas seulement un sens religieux : la péroraison place sur la scène publique et politique le moment religieux de la prise de voile, dont toute la responsabilité est attribuée à la postulante elle-même, comme le montre la deuxième personne du pluriel sujet («Enveloppez-vous»). En définitive, quoique Louise de la Miséricorde soit désormais « connue de Dieu seul » (Bossuet, 1926b, p. 58), le triomphe politique de l'Église est désormais complet. La péroraison entre en tension avec l'exorde adressé à la reine ; cette tension énonciative rend impossible à l'ancienne maîtresse royale de « vivre cachée [...] à tout le monde » (Bossuet, 1926b, p. 58).

\section{Conclusion}

" Mais la religion elle-même n'est-elle pas nécessairement liée à l'ordre public ? Elle tombe ou s'affoiblit avec lui » (Massillon, 1835, p. 594). Certes, cette phrase de Massillon résume avec précision et concision l'idée que, si le discours religieux se fonde sur la spiritualité et la morale, il n'est pas concevable de l'extraire complètement du champ politique et social. L'utilisation des motifs de la vanité et du dégoût du monde dans le Carême du Louvre et le Sermon pour la profession de La Vallière fonctionnent ainsi. Faire triompher la morale chrétienne suppose que le discours religieux soit actif sur la scène d'énonciation politique. La topique de la vanité est reconfigurée : elle n'appartient plus seulement au dogme catholique, mais devient paradoxalement une arme politique pour le prédicateur. Du reste, le rejet du monde auquel se livre La Vallière en entrant au Carmel se charge d'un sens résolument public : elle devient une attraction que l'on vient contempler. Ce dénouement de l'affaire, aussi paradoxal soit-il, et létude de l'énonciation révèlent que le mépris des biens de ce monde ne saurait en aucun cas se passer du monde.

\section{RÉFÉRENCES}

Bossuet, J. B. (1926a). CEuvres oratoires de Bossuet. T. 4. Éd. critique de l’abbé J. Lebarcq, revue et augmentée par Ch. Urbain et E. Levesque. Paris, France : Desclée De Brouwer et $C^{\text {ie }}$. 
Bossuet, J. B. (1926b). CEuvres oratoires de Bossuet. T. 6. Éd. critique de l'abbé J. Lebarcq, revue et augmentée par Ch. Urbain et E. Levesque. Paris, France : Desclée De Brouwer et $C^{\text {ie }}$.

Bossuet, J. B. (1927). Euvres oratoires de Bossuet. T. 3. Éd. critique de l'abbé J. Lebarcq, revue et augmentée par Ch. Urbain et E. Levesque. Paris, France : Desclée De Brouwer et $C^{\text {ie }}$.

Bourdieu, P. (1971). Genèse et structure du champ religieux. Revue française de sociologie, 12(3), 295-334. Récupéré de http://www.persee.fr/doc/rfsoc_0035-2969_1971_num_12_3_1994

Cagnat-Debœuf C. (2001). Préface. Dans J. B. Bossuet Sermons. Le Carême du Louvre (p. 7-49). Paris, France : Gallimard.

Joly, A. (2011). L'article, instrument de modalisation chez Gustave Guillaume (1919). Modèles linguistiques, 64, 103-115. Récupéré de http://journals.openedition.org/ml/355

Maingueneau, D. (2004). Le Discours littéraire. Paratopie et scène d'énonciation. Paris, France : Armand Colin.

Massillon, J. B. (1835). Sermon sur les écueils de la piété des grands. CEuvres de Massillon. T 1. Paris, France : Lefèvre.

RÉSUMÉ : Nous voulons analyser les motifs de la vanité et du mépris du monde dans le Carême du Louvre et dans le Sermon pour la profession de La Vallière dans une perspective à la fois topique et énonciative. La liaison de Louis XIV et de La Vallière pose un double problème religieux et politique. À cet égard, Bossuet utilise la topique de la vanité comme une arme paradoxale qui montre que le mépris du monde ne peut se passer du monde. Le champ religieux et le champ public se reflètent alors sans cesse l'un dans l'autre.

Mots-clés : Bossuet, sermon, rhétorique, énonciation, vanité

\section{Bossuet versus La Vallière: the motives of vanity, between religious and political issues}

ABSTRACT: We will analyse the motives of vanity and of contempt of the world in Bossuet's Carême du Louvre and Sermon pour la profession de La Vallière in both a topical and clearly enunciated perspective. The relationship between Louis XIV and La Vallière raises a religious and political issue: the motive of vanity is used by the preacher not only as a theological argument, but also as a political weapon. The motives of contempt of the world can't do without the world itself: religious and political fields are constantly reflected on one other.

Keywords: Bossuet, homily, rhetoric, enunciation, vanity 